\title{
FLEXO.IN-FORM. Laminary envelopes to active flexion through geometric-material optimization processes
}

\author{
Mauro Chiarella \\ Consejo Nacional de Investigaciones Científicas y Técnicas, Universidad Nacional del Litoral \\ | Argentina | mchiarella@hotmail.com \\ Ma. Luciana Gronda \\ Universidad Nacional del Litoral | Argentina | grondaluciana@hotmail.com \\ Martín W. Veizaga \\ Universidad Nacional del Litoral | Argentina | veizagamartin@hotmail.com
}

\begin{abstract}
Flexo.In-Form is a prototype derived from an experimental exercise to verify the structural effort of active flexion. Bending behavior is used as a design tool applied to structures that base their geometry on the elastic deformation of flat elements. Through "Integrative Processes" and a "Performance-Oriented Design Approach", the operational relationship between active mechanical mechanisms, material performance and geometric design has been enhanced. The proposed geometric and material optimization process extends the experiences with physical models of complex shapes through computational numerical calculation and its possibilities of simulation and digital evaluation.
\end{abstract}

Keywords: Performance; Form-finding; Parametric Design; Physical Simulation; Digital Manufacturing.

\section{INTRODUCCIÓN}

Se presenta el proceso de ideación, fabricación y montaje del prototipo Flexo.In-Form el cual instrumentaliza la deformación elástica de materiales laminares para la autoconformación de estructuras curvas complejas. Revindica la histórica técnica del 'form-finding' como antecedente metodológico para la exploración de la forma compleja y la amplifica digitalmente mediante la implementación de procesos integradores (PI) concentrados en abordar los procesos de ideación y construcción desde un enfoque de diseño orientado al desempeño (EDOD). La implementación de PI constituye nuevos procedimientos y formas de abordaje para repensar los procesos de ideación y abre múltiples posibilidades para emancipar del paradigma moderno- la condición material en arquitectura ya que introducen un nuevo nivel de integración en diseño arquitectónico.

La investigación propone, por un lado, alternativas a los modos de producción del mundo material derivados del modelo industrial (si bien por cuestiones de factibilidad se recurre a materiales estandarizados para la fabricación, los mismos son resignificados mediante el diseño geométrico de componentes arquitectónicos complejos y el aprovechamiento de sus propiedades intrínsecas para el desempeño estructural), y por otro lado, una metodología capaz de revertir la división metodológica con la que se abordan los procesos "convencionales" de proyectación (representación, cálculo y construcción) mediante la implementación de PI y el desarrollo de un algoritmo de diseño paramétrico orientado a mejorar la relación operacional entre: procesos de optimización geométrica, aprovechamiento de las propiedades del material y desempeño estructural.
El objetivo es verificar las ventajas estructurales que pueden ser generadas mediante flexión activa (FA) en envolventes laminares curvas complejas accesibles en las condiciones de posibilidad detectadas en contextos heterogéneos de producción y construir una mirada crítica sobre el avance de los procesos computacionales en arquitectura.

\section{CONTEXTUALIZACIÓN}

En Latinoamérica y más precisamente en Argentina, los avances convergentes a la línea de investigación propuesta operan condicionados por cierta inercia disciplinar ligada al paradigma productivo industrial y en contextos heterogéneos de producción que reúnen procesos artesanales o pre-industriales; mecánicos 0 industriales y digitales o pos-mecánicos, en menor medida. Por un lado, el alto grado de producción artesanal mantiene su inercia tanto por cuestiones socioeconómicas -vinculadas a los elevados costos de capacitación de la mano de obra requerida- como así también por factores culturales fuertemente arraigados a la tradición histórica de la disciplina. Por otro lado, la falta de desarrollo científico acorde a estos temas, vinculada a la necesidad de mayor financiamiento para abordar, producir y montar experimentaciones materiales a escala arquitectónica indica que el problema de la escala en arquitectura representa los mayores desafíos en cuanto a desarrollo científico aplicado y amerita trabajos que se aborden científicamente en esta dirección.

En relación con el análisis de las posibilidades de acceso a los materiales, métodos e instrumentos, estudios previos determinaron que la FA reúne ampliamente las condiciones de viabilidad determinantes para la concreción práctica a partir de la experimentación directa con herramientas de fabricación CNC (corte 2D) y materiales 
laminares estandarizados y ampliamente comercializados, por lo tanto, disponibles y de bajo costo.

\section{ENFOQUE TEÓRICO-METODOLÓGICO}

La necesaria especificación del comportamiento material revindica las históricas técnicas del 'form-finding' como antecedente metodológico para la exploración formal en directa relación al desempeño estructural. La experimentación con modelos físicos para la concepción de la forma compleja es amplificada mediante la implementación del cálculo numérico computacional y sus posibilidades de simulación y evaluación digital habilita operar mediante un EDOD (Gronda y Chiarella, 2017).

\section{FLEXIÓN ACTIVA}

La FA consiste en la instrumentalización de la deformación elástica como capacidad del material para estabilizarse y auto-conformarse (Lienhard, 2014) y se propone como alternativa de diseño y construcción -no como una tipología estructural predefinida-

El trabajo aplicado a estructuras que basan su geometría en el aprovechamiento de la deformación elástica-activa de materiales laminares -inicialmente planos- abre nuevas complejidades para el proceso de diseño y construcción de envolventes arquitectónicas curvas complejas. Avances previos dan cuenta de que esta lógica proyectual brinda aportes confiables a través de la interacción de variables relacionales parametrizadas (La Magna, Schleicher and Knippers, 2016).

Si bien la máxima en ingeniería es limitar la cantidad de flexión en las estructuras, este enfoque en realidad aprovecha la flexión para la creación de diseños complejos y extremadamente livianos. Las placas de multilaminado de madera de bajo espesor rara vez son utilizadas como estructura principal en arquitectura debido a su baja rigidez a la flexión. Sin embargo, muchos materiales laminares como madera contrachapada, metales, plásticos y polímeros reforzados con fibra no solo son flexibles, sino que también tienen una alta resistencia a la tracción. Estas dos propiedades juntas reúnen grandes potenciales para las estructuras a FA porque permiten que los elementos sufran grandes deformaciones elásticas y resistan tensiones elevadas antes del fallo (Schleicher et al.,2015).

Frente a la dificultad de evaluar el comportamiento de estas estructuras y anticipar con precisión su geometría deformada se recurre a la construcción de modelos físicos y ensayos empíricos para el reajuste de las restricciones de diseño en la definición paramétrica.

\section{'FORM-FINDING' ANALÓGICO + DIGITAL}

La retroalimentación de la información en procesos de 'form-finding' analógico+digital constituye en sí la metodología operacional desde la cual abordar un EDOD.

En principio, el 'form-finding' analógico es la determinación escalar de la forma a través de la replicación de las condiciones materiales y contextuales del mundo real, constituye un método bien establecido en arquitectura e ingeniería, y si bien, hay una larga historia de experimentación en este campo -apoyada por investigaciones científicas y ejemplos construidos incluso antes del uso generalizado del diseño computacional en arquitectura-, continúa aportando una lectura esencial para la innovación e investigación en diseño.

El 'form-finding' analógico+digital -como estrategia metodológica de aproximación, búsqueda y optimización de la forma compleja- declara cierta continuidad con los modelos paramétricos físicos del S.XX, emprendidos igualmente por arquitectos e ingenieros, pero avanza en rigurosidad y precisión técnica digitalizando una práctica disciplinar histórica a partir de PI. En publicaciones anteriores se presentan y analizan casos de estructuras a $F A$, sus desarrollos materiales, métodos y enfoques (Gronda, Chiarella, Veizaga, 2017).

\section{PROCESOS INTEGRADORES}

Los PI ('Integrative Approach', Lienhard et al., 2013) de ideación y fabricación digital en arquitectura potencian y enriquecen las posibilidades instrumentales, metodológicas y técnico-culturales con las que cuenta la arquitectura.

En términos instrumentales, operar desde PI implica: modelado paramétrico -de lógica matemático algorítmica; simulación digital -para la predicción del comportamiento físico-; evaluación de cálculo numérico computacional y finalmente, fabricación digital.

Los PI habilitan lógicas proyectuales orientadas a revertir la división metodológica inherente a los procesos "convencionales" de proyectación. Reposiciona a los diseñadores en un papel estratégico para la construcción de modelos 'científicos', fórmulas paramétricas 0 algoritmos de diseño in-formados con los datos obtenidos de un proceso de lógica continua e iterativa que reúne las distintas instancias del proceso proyectual y su concreción. Redefine el rol del arquitecto en el proceso mismo de la búsqueda de la forma, amplía el campo de acción y exige considerar simultáneamente las diferentes dimensiones o variables que operan promoviendo un abordaje integrador entendido como EDOD.

\section{METODOLOGÍA}

Flexo.In-Form propone abordar el diseño y la construcción desde PI con EDOD al utilizar el comportamiento a la flexión como herramienta de ideación para la experimentación formal y la construcción de superficies continuas de forma libre (free-form) a partir de elementos de curvado simple -desarrollables- mediante un proceso de construcción y montaje sencillo.

La construcción de la envolvente laminar a escala arquitectónica parte del diseño de una estructura tramada de doble curvatura en forma de rejilla laminar o "grid-shell" de resistencia multidireccional.

El prototipo utiliza láminas planas que presentan posibilidades de deformación limitadas ya que se deforman principalmente a lo largo del eje de inercia más débil y, por lo tanto, no pueden adaptarse fácilmente a geometrías complejas o de doble curvatura. Tomando esta limitación geométrica como una ventaja especial en términos de diseño, se instrumentaliza la deformación elástica del material potenciándolo a partir del diseño de su geometría y maquinaria CNC para su fabricación. 


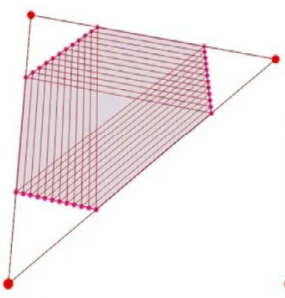

(a)

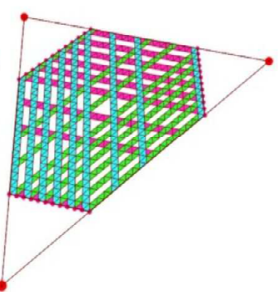

(b)

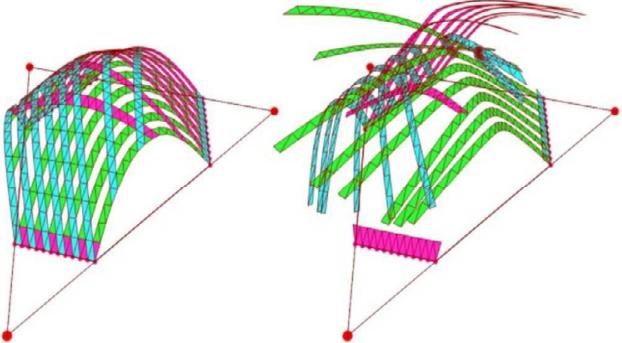

(c)

(d)

Figura 1: Geometría inicial en Grasshopper (a); construcción de cintas coplanares a partir de los puntos obtenidos de la subdivisión de las 3 líneas de apoyo (b); Simulación digital de los esfuerzos físicos a flexión de las cintas con Karamba3D (c) y un-roll de cintas para fabricación CNC (d).

A los fines de explicar el PI desde el que se opera, se definen 3 instancias de ideación: Modelado paramétrico, simulación y evaluación digital: Forma plana inicial; Forma Deformada (a flexión) y forma Calibrada/Optimizada, respectivamente. Por su parte, el proceso de fabricación y montaje también es organizado en otras 3 instancias: corte, replanteo y organización de las cintas (a); 'izamiento' de la forma relajada (b) y instrumentalización de la flexión para la auto-conformación de la envolvente laminar y tensionado de la forma final (c). Esta división metodológica recupera su enfoque integrador en la interacción entre una instancia y las demás (Figuras 2 y 3 ).
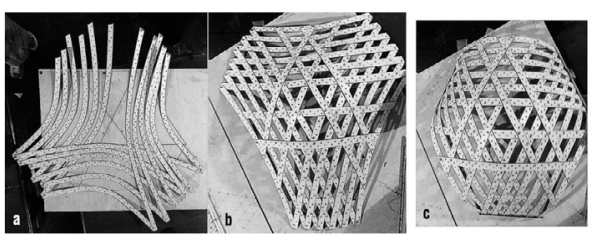

Figura 2: (Prototipo 1:10) Corte 2D y organización de componentes laminares (a); unión de los componentes e "izamiento" (b); instrumentalización de la flexión para la autoconformación de la envolvente laminar (c).

La sincronización de los estudios físicos y digitales debe garantizar que las técnicas de simulación para la búsqueda de la forma proporcionen una descripción precisa del comportamiento real del material y, al mismo tiempo, permitan re-in-formar a la geometría resultante de la estructura.
Con el reajuste de la geometría inicial se mejora el desempeño de la estructura garantizando que las tensiones permanezcan dentro del rango de trabajo permitido por el material.

Los prototipos construidos a escala permiten corroborar empíricamente las fuerzas a las que puede ser sometido el material en el espesor seleccionado. De los ensayos físicos se determinan radios de curvatura, mínimos y máximos, antes de que se produzca la rotura. Dichas limitaciones se integran como datos de diseño en la definición paramétrica (Grasshopper) y se utilizan para calibrar las simulaciones digitales de los esfuerzos físicos a flexión (Karamba3D versión 1.3.3. PRO).

\section{DESARROLLO}

Inicialmente, las cintas planas se flexionan/curvan mediante el motor de simulación física: Karamba3D para obtener una envolvente estructural ('grid-shell') de autoformación que luego es calibrada y desarrollada (un-roll) para habilitar su fabricación con herramientas CNC.

\section{MODELADO PARAMÉTRICO}

El algoritmo se construye inicialmente a partir de un triángulo base cuyos vértices se pueden adaptar a cualquier topología. Dentro del mismo se inscribe un hexágono de donde se individualizan sus 6 segmentos: 3 líneas para construir el anclaje y 3 líneas para construir los arcos a flexión.

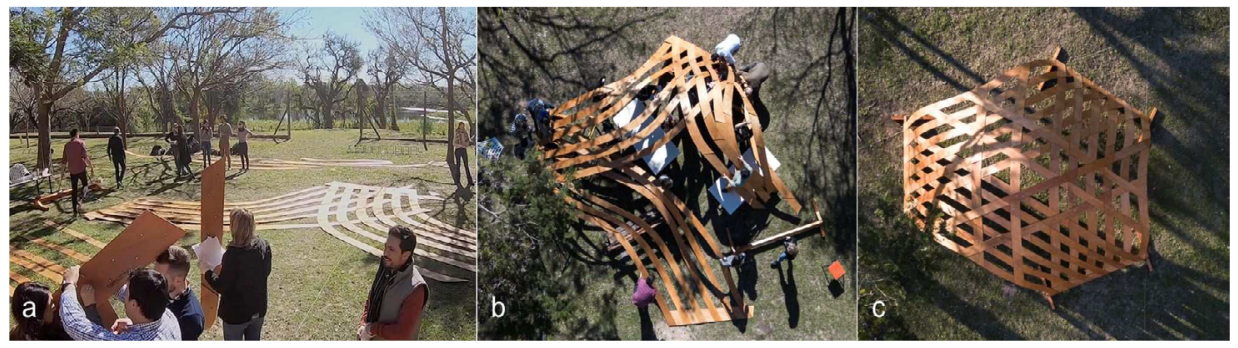

Figura 3: Metodología de montaje del prototipo escala 1:1: corte 2D de componentes laminares, replanteo y organización de las cintas (a); unión de los componentes e "izamiento" de la envolvente laminar (b); Instrumentalización de la flexión para la auto-conformación de la envolvente laminar (c). (https://vimeo.com/360822874) 
La subdivisión de las 3 líneas de anclaje en "x" segmentos iguales otorga los puntos de apoyo y su interconexión genera la grilla triaxial base que conforma las cintas del modelo como mallas trianguladas (Figura 1a). El orden topológico en la descripción paramétrica de la malla permite identificar el contacto entre las cintas superpuestas y que en la simulación a la flexión las mismas se desplacen juntas (Figura 1b), funcionando como una red ('grid-shell) en sentido físico.

Para materializar el mismo comportamiento estructural simulado en el modelo digital los prototipos físicos se construyen mediante uniones fijas en los cruces de las cintas (Figura 4).

\section{SIMULACIÓN Y EVALUACION DIGITAL}

Las cintas se flexionan en interacción con la intensidad de la fuerza aplicada y la resistencia elástica del material (madera de guatambú laminada de $3 \mathrm{~mm}$ en 3 capas) formando en conjunto una trama geométrica estructural de doble curvatura que es construida por cintas 0 componentes laminares de curvatura simple (Figura 1c), por lo tanto, desarrollables para su fabricación digital con tecnología de corte CNC. (Figura 1d)

En ambas instancias de simulación y evaluación se trabajan con el plug-in: "Karamba3D" (versión 1.3.3. PRO).

EI EDOD analizó el estudio de tensiones y radios de curvatura en relación con la cantidad de cintas de refuerzos convenientes para la fabricación y montaje 1:1 (Figura 4).

En el proceso de 'form-finding' digital y para la generación de la forma de la envolvente, a la trama digital inicialmente plana se le aplica una fuerza de tipo gravitacional "Z" (normal al triángulo base). Para el anclaje de la geometría se fija el desplazamiento $x, y, z$ de los puntos de apoyo y se permite la rotación $x, y, z$ de las cintas sobre esos puntos, dejando que la geometría solo experimente una deformación de curvatura que la flexión del material permita dentro de las variables definidas (material, fuerzas y resistencias actuantes).
La malla resultante es sometida a un análisis de curvatura (Gaussiana, radio mínimo y radio máximo) y de stress de von mises, lo que permite localizar rápidamente los sectores más relajados y más tensionados respectivamente en toda la geometría resultante (Figura 5).
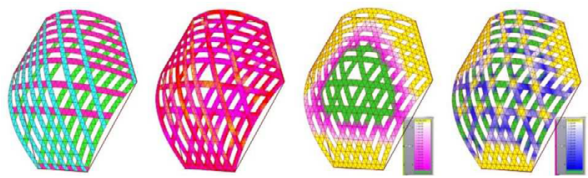

Figura 5: Simulación digital a flexión, Análisis de Curvatura Gaussiana y Van Mises Stress.

Con los valores de radio mínimo y radio máximo se pudo ensayar prototipos materiales en distintas escalas que representan el grado de tensión del material (flexión activa) en distintas partes de la geometría y permitió predefinir una escala y espesor de material para el prototipo 1:1.

El proceso de optimización de la sección transversal del material, dentro de la definición de grasshopper, utiliza el componente de Karamba 3d: "OptiCroSec" (Cross Section Optimizer), el cual permite calibrar espesor y resistencia material en relación con las deformaciones locales de la 'grid-shell'. Las cintas se readaptan relajando la curvatura en los sectores más tensionados y tensionando sectores de la malla más relajados. Este proceso readapta la forma de la malla y conduce a una redistribución de las tensiones internas a valores más próximos entre máximos y mínimos, reduciendo la probabilidad de falla local en sectores muy flexionados o relajados (Figura 6).

\section{FABRICACIÓN DIGITAL}

El proceso de fabricación implica atender -desde las instancias iniciales de ideación- a la escala, al tamaño de los componentes, a las medidas comerciales de los materiales laminares disponibles en el mercado y al tamaño de corte máximo admitido por la herramienta CNC seleccionada para la producción.
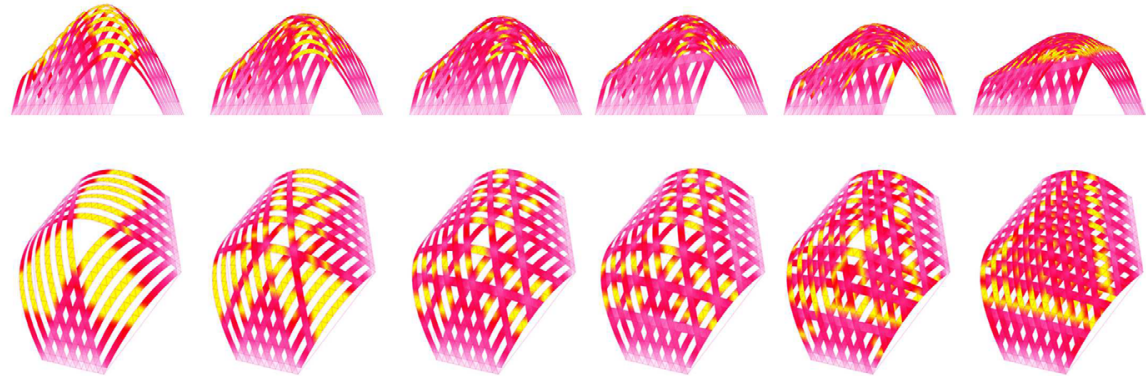

Figura 4: El modelo de la izquierda muestra una estructura poco tramada donde las cintas trabajan independientemente unas de otras concentrando la curvatura y las tensiones de deformación en el tramo central. El modelo de la derecha, muestra una estructura tramada al $100 \%$ de sus posibilidades geométricas logrando una alta resistencia de cascara, pero concentrando las tensiones en un área muy estrecha y lineal (amarillo). Los esquemas centrales muestran tensiones distribuidas y menos localizadas y las zonas de mayor tensión mantienen una doble curvatura aumentando la resistencia. 


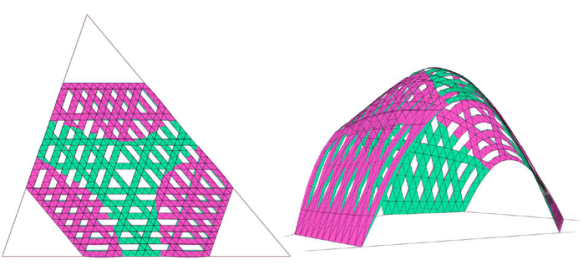

Figura 6: Esquema de variación. El Verde muestra la forma deformada Inicial y el magenta la Forma Optimizada, donde se puede visualizar la relajación de la curva en la parte superior y el tensionado en los sectores de apoyo.

En un $\mathrm{PI}$ la definición paramétrica asocia todas las acciones y restricciones de diseño en relación a la escala para la fabricación. El componente "Un-Roll" de Kangaaro desenrolla las cintas, luego el "Orient" las abate al plano X, Y; "Split" las fragmenta en partes cuyo máximo es el tamaño de la mesa de corte y "Rotate" gira las partes en el sentido de la fibra del material utilizado, otorgando continuidad de resistencia elástica a flexión en toda la cinta y luego "Nesting" las anida minimizando desperdicios materiales.

Previo a la fabricación y montaje del prototipo Flexo.InForm se ensayaron dos modelos en karical de 0.06 y de $0.08 \mathrm{~mm}$, en escalas $1: 10$ y $1: 5$ respectivamente, testeando la relación más conveniente entre geometría, material y comportamiento. Los prototipos experimentales no eran aún lo suficientemente representativos para el paso a la escala 1:1, principalmente en relación a la estabilidad de la forma global. En el cambio de escala es cuando la posibilidad de reajuste del modelo digital se vuelve relevante. La asociación paramétrica de las variables geométricas, las propiedades y espesor del material y el desempeño del conjunto evaluado digitalmente, pero los ajustes necesarios para afrontar el cambio de escala (Figura 7).

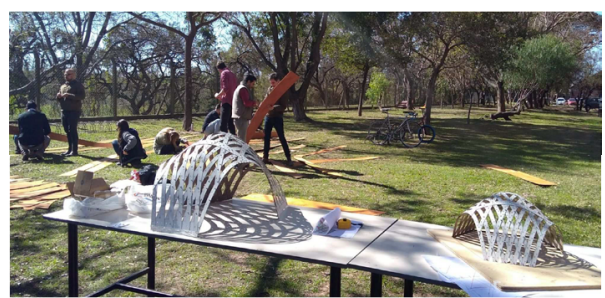

Figura 7: Prototipos a escala 1:10 y 1:5 utilizados como referencia en la instancia del armado 1:1.

Finalmente, siendo el contrachapado altamente idóneo para grandes deformaciones a flexión por su relativo bajo módulo elástico, el modelo digital en escala 1:1 se ajustó para ser fabricado en multilaminado fenólico de $3 \mathrm{~mm}$ de guatambú en 3 capas. Las 2 capas exteriores estructurales y la central de relleno.

\section{MONTAJE MANUAL}

Desde las primeras instancias de ideación, el montaje de la envolvente laminar de auto-formación implicó considerar especialmente al problema de la escala, ya no en función de las dimensiones de los componentes, de las propiedades y espesores de los materiales o las herramientas $\mathrm{CNC}$, sino en relación con el peso, la cantidad y dimensión de los componentes y de los recursos humanos y operativos, necesarios y disponibles.

Siendo la instancia más empírica del proceso, para el montaje se realizó un instructivo detallado para la ubicación, el replanteo y el ensamble de los componentes de cada cinta con la debida codificación. Los distintos componentes se ensamblan entre sí con uniones fijas en los solapamientos conformando las cintas continuas, las cuales aisladas posen muy baja resistencia elástica como para soportar un arco fijado en solo dos apoyos. El fijado del entrecruzamiento de cintas se realiza con tornillos de 4 $\mathrm{mm}$. El entramado le confiere a la 'grid-shell' una capacidad de redistribución de cargas. (Figura 8).

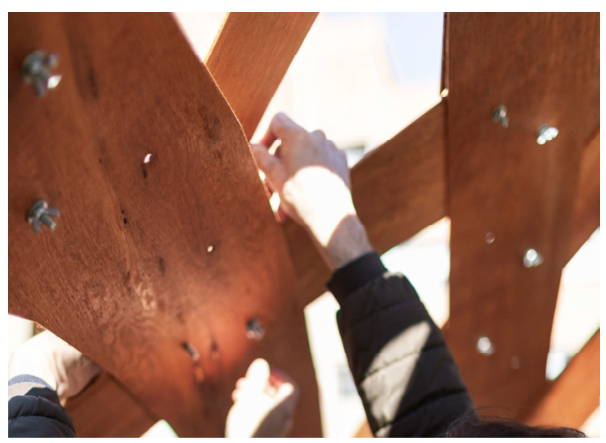

Figura 8: Uniones fijas mediante tornillos en la instancia de montaje 1:1.

El tensionado de la forma final, que implicó llevar a su lugar de replanteo cada uno de los 3 apoyos de la estructura, le imprime a la forma la tensión necesaria y suficiente para que la flexión auto-conforme la forma diseñada.

El proceso de replanteo, "izamiento" y conformación a la flexión del prototipo se realizó en aproximadamente 3 horas y con un grupo de 25 alumnos del Módulo: Proyectación y Fabricación Digital: PyFD-2019, propuesta académica de la Maestría en Arquitectura FADU-UNL.

El montaje de Flexo.In-Form o prototipo en escala 1:1 exhibió los temas abordados en las etapas anteriores: diseño; simulación; análisis y fabricación , como así también, cada una de las variables relacionales: geometría; material; estructura; resistencia; peso manipulación y desempeño, otorgando conclusiones que formarán parte de futuras parametrizaciones para una mayor precisión o rigor científico, pero, fundamentalmente, se validó la utilidad de los PI como propuesta metodológica para el reajuste de un próximo modelo/prototipo (Figura 9).

\section{RESULTADOS Y DISCUSIONES}

El diseño, construcción y montaje de Flexo.In-Form permitió analizar las ventajas estructurales que pueden ser generadas mediante la deformación elástica en envolventes laminares a FA habilitando construir una mirada crítica sobre el avance de los procesos computacionales en arquitectura. Sobre la base de las estrategias formales e investigación proyectual con PI, la construcción de un modelo paramñetrico de simulación digital aporta vistas previas confiables para envolventes arquitectónicas curvas a través de la interacción de 


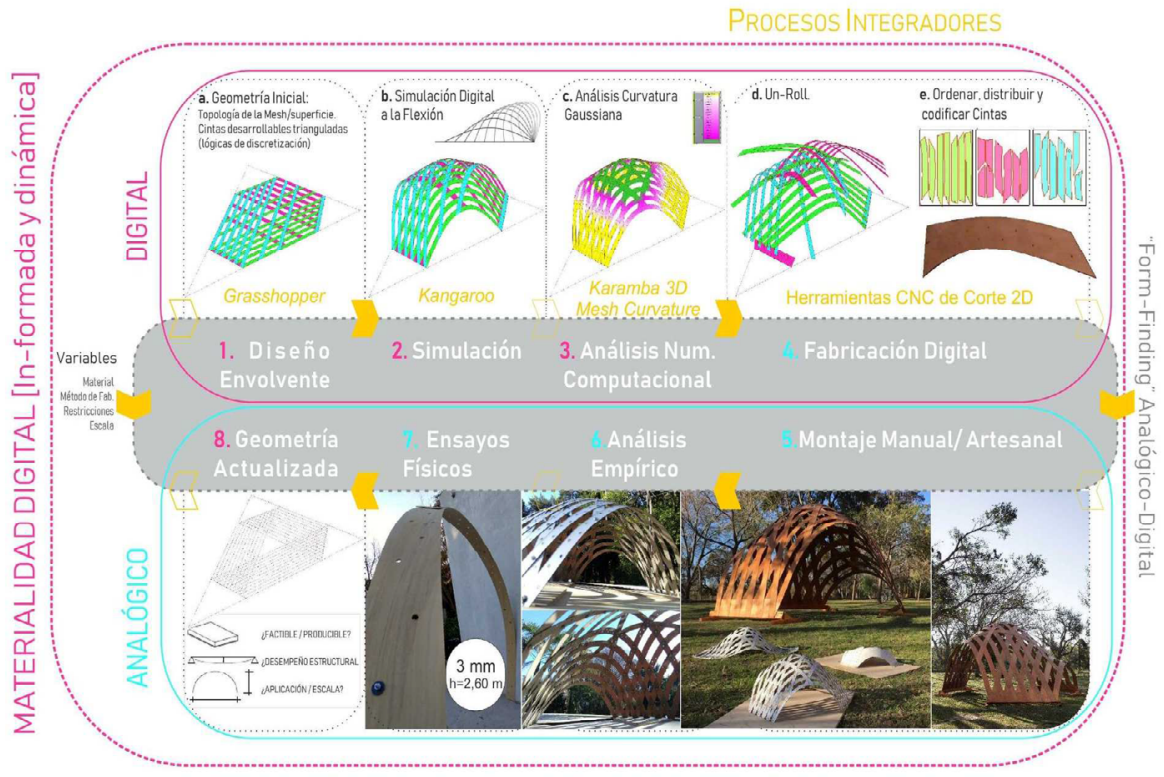

Figura 9: Cuadro de autoría propia sobre la metodología aplicada con P.I

variables relacionales parametrizadas. El análisis de la curvatura gaussiana expone las zonas de la envolvente con doble curvatura permitiendo controlar la torsión que supone en las cintas. El análisis del radio de curvatura máximo y mínimo identifica el componente -y el sector del componente- más y menos curvado posibilitando adaptar la geometría a los rangos admisibles y/o deseables para la flexión del material.

La experiencia verifica que la metodología propuesta como EDOD permite orientar los procesos de diseño a la optimización de los recursos. La integración estratégica de modelos de simulación físico-geométrica sumada a la herramienta metodológica del form-finding, analógicodigital, amplía el espacio de diseño y las posibilidades de construcción de envolventes laminares curvas complejas mediante la discretización geométrica para la fabricación de componentes a partir de materiales laminares ampliamente disponibles en el mercado.

La instancia de simulación y evaluación digital permite una mayor precisión a la hora de seleccionar y definir los materiales, las formas, los espacios y las tecnologías, minimizando así las decisiones basadas únicamente en la definición de categorías tipológicas estructurales, tradición local o experiencia directa. Aun así, el mayor reto continúa siendo dominar los datos de la simulación para cuantificar y analizar el esfuerzo real de las tensiones a flexión en el cálculo estructural y contribuir a un modelo científico más riguroso, tanto de la forma global como de las características locales de los componentes constitutivos de las cintas, en las cuales, las características geométricas y las propiedades del material son inevitablemente relacionales y afectan al desempeño estructural.

En este sentido la retroalimentación de los datos obtenidos de los ensayos físicos con prototipos a escala resulta clave para realizar los reajustes necesarios que informan la definición paramétrica en la instancia de simulación y análisis digital. La integración de las restricciones de diseño -tanto geométricas como materiales- para el mejoramiento del comportamiento estructural, obtenidas de la retroalimentación en procesos de 'form-finding', analógico-digital, constituye un enfoque de gran potencial para muchas aplicaciones.

\section{REFERENCIAS}

Gronda, L., Chiarella, M. y Veizaga, M. (2017). "Diseño orientado a la optimización en envolvente laminares de flexión activa". Revista ARQUITECNO X. Ediciones ITDAHu. Resistencia. vol. 1 p. $48-57$.

Gronda, L., Chiarella, M., y Veizaga, M. (2019) «Flexo.InForm.2019. Manufactura digital y optimización geométrica mediante programación visual». Polis, n 16. FADU.UNL

Gronda, L., y Chiarella, M. (2017). "Materialidad Digital. Análisis de estrategias performativas transferibles al diseño resiliente". SIGraDi 2017 Proceedings of the 21th SIGraDi Conference. Chile, Concepción 22 - 24 November 2017, pp.51-59.

La Magna, R., Schleicher, S., \& Knippers. J. (2016) "BendingActive Plates. Form and Structure". In: R. La Magna, J. Knippers, S. Adriaenssens, F. Gramazio, M. Kohler, A. Menges, \& M. Pauly (eds.): Advances in Architectural Geometry. Pag. 170-186.

Lienhard, J. (2014). Bending Active Structures, Form-Finding Strategies Using Elastic Deformation in Static and Kinematic Systems and Structural Potential Therein, Ph.D. Thesis, ITKE, University Of Stuttgart.

Lienhard, J., Alpermann, H., Gengnagel, C., \& Knippers, J. (2013). "Active Bending, A Review on Structures where Bending is used as a Self-Formation Process". In: International Journal of Space Structures.

Schleicher, S., Rastetter, A., La Magna, R., Schönbrunner, A., Haberbosch, N., \& Knippers, J. (2015). Form-Finding and Design Potentials of Bending-Active Plate Structures. 